\title{
Anatomía versus fisiología coronaria para guiar las intervenciones en la enfermedad coronaria crónica
}

\author{
Alejandro Martínez \\ Pontificia Universidad Católica de Chile
}

A pesar de los avances en su prevención y tratamiento, la enfermedad coronaria continúa siendo una de las principales causas de muerte en el mundo. Esto se debe a su alta prevalencia y a la incierta evolución de las lesiones crónicas hacia los síndromes coronarios agudos, que determinan el riesgo por infarto del miocardio o muerte súbita. Anatómicamente estas lesiones pueden permanecer estables, incluso regresar, o progresar en forma lenta o rápida, con o sin síntomas. Por lo tanto la estratificación de riesgo en los pacientes con enfermedad crónica es esencial para guiar las decisiones terapéuticas. En este sentido, existe amplia controversia sobre si el grado de estenosis, sus consecuencias fisiológicas o la morfología de las placas coronarias, sería el mejor determinante de riesgo. Esta discusión es especialmente relevante cuando queremos realizar intervenciones de revascularización útiles para disminuir el riesgo.

La decisión es más sencilla en los pacientes con síndromes coronarios agudos, con elevación de troponinas, porque en ellos las terapias de revascularización categóricamente reducen la mortalidad e infarto ${ }^{1-2}$. Sin embargo, en los pacientes con enfermedad coronaria crónica, aunque se ha demostrado mejoría de la angina, en muchos casos persisten dudas sobre los beneficios de la revascularización en la reducción de eventos mayores. Varias décadas atrás, aunque marginalmente, se comprobó que los pacientes que se beneficiaban con cirugía coronaria eran los que tenían una enfermedad muy extensa y grandes áreas de miocardio amenazado, como ocurre en enfermedad de tronco común izquierdo, 3 vasos y posiblemente enfermedad de 2 vasos con compromiso de la descendente anterior proximal ${ }^{3}$. La presencia de isquemia en los test de provocación también identificaba a los pacientes que reducían su mortalidad con cirugía ${ }^{3}$. Sin embargo, estos datos fueron válidos con anterioridad al manejo médico contemporáneo. Actualmente usamos fármacos como estatinas, inhibidores del eje renina-angiotensina-aldosterona y antiplaquetarios, que individualmente reducen el riesgo de infarto y muerte, y no se usaban en esa época. Tampoco se tenía certeza que los cambios de hábitos que hoy reconocemos importantes, como la suspensión del tabaquismo y el ejercicio físico, mejoran el pronóstico ${ }^{4}$. Es decir, fueron válidos previo a la implementación de lo que hoy llamamos terapia médica óptima (TMO). 
En la actualidad, en cambio, el rol de las intervenciones en pacientes con enfermedad coronaria crónica estable, basada en anatomía o isquemia, salvo excepciones como enfermedad del tronco común izquierdo (que no se cuestiona) o enfermedad severa de 3 vasos, apoyada solo por registros contemporáneos ${ }^{5}$, en la mayoría de los casos ha sido cuestionado. En 2 estudios randomizados recientes las tasas de muerte e infarto observadas en lo pacientes tratados con TMO con o sin angioplastía en el COU$\mathrm{RAGE}^{6} \mathrm{y}$, con o sin angioplastía o cirugía en el BARI $2 \mathrm{D}^{7}$, fueron similares. A estos estudios, sin embargo, se les reprocha 2 aspectos. Por un lado, cuando se hizo angioplatía se usó casi exclusivamente stents sin drogas, lo que se asocia a mayor riesgo de eventos que cuando se implantan stents medicados, especialmente de segunda generación. Y, por otro, se critica que la magnitud de la isquemia no era muy importante. De hecho, en el COURAGE solo $33 \%$ de los pacientes tenía isquemia de $10 \%$ o más, valor que se estima genera el cambio de riesgo.

Numerosos estudios observacionales antiguos sugirieron fuertemente que la extensión de la isquemia, evaluada con distintos métodos, se correlaciona con riesgo de muerte e infarto, lo que podría reducirse con revascularización $^{8-9-10-11}$. Asimismo, hubo estudios randomizados que apoyaron el beneficio de esta intervención cuando existe isquemia extensa ${ }^{12-13}$. Pero, otra vez, estos datos fueron obtenidos antes de la TMO. Los estudios recientes, sin embargo, aunque con datos observacionales, no apoyan la isquemia para justificar revascularización. Se publicaron sugerencias desde el COURAGE, que incluye TMO, que favorecían la angioplastía cuando la isquemia era moderada o severa, pero cuando se ajustaron los pacientes por sus características basales estas diferencias ya no fueron válidas ${ }^{14}$. Es más, en un estudio alejado del COURAGE, en 621 pacientes la isquemia basal no se asoció a mortalidad, infarto o síndrome coronario agudo en un seguimiento promedio de 4,7 años, mientras que la extensión de la enfermedad angiográfica si se asoció a eventos ${ }^{15}$. Similarmente en un análisis del BARI-2D, que evaluó el impacto pronóstico del SPECT, no se demostró mayor riesgo de muerte o eventos cardiovasculares, según la magnitud de la isquemia ${ }^{16}$. También en el estudio STICH fue la magnitud de la ateroesclerosis y no la isquemia lo que identificó a los pacientes de mayor riesgo alejado $^{17}$. Se suma a lo anterior el registro internacional CONFIRM, de más de 23.000 pacientes sometidos a angio-TAC coronario, que resalta el número de vasos con estenosis $>50 \%$ y no la isquemia como el mayor predictor de riesgo ${ }^{5}$.
Otro test de isquemia como la medición de Reserva de Flujo Fraccional (FFR) en el laboratorio de hemodinamia, como comenta el Dr. Aninat en esta revista, ha alcanzado amplia aceptación en la comunidad cardiológica, para guiar las intervenciones. Esto se basa en la asociación de un FFR bajo con isquemia significativa ${ }^{18}$ y principalmente por los hallazgos del estudio FAME-2 ${ }^{19}$. En este estudio, realizado en pacientes con enfermedad coronaria crónica, la angioplastía coronaria en lesiones con FFR bajo reducía significativamente los eventos comparado con el tratamiento médico óptimo (TMO). Sin embargo, la intervención en lesiones con FFR $<0,8$ solo redujo la necesidad de nuevas intervenciones y no hubo disminución de infarto o muerte.

Esta serie de datos ha hecho pensar que eventualmente la detección de isquemia en los estudios de provocación es un marcador de la presencia y severidad de la enfermedad coronaria, siendo esto último y no la isquemia lo que confiere el riesgo de eventos adversos. Este concepto puede soportarse por nuestro actual entendimiento de la fisiopatología de los eventos coronarios agudos ${ }^{20}$. Estos eventos surgen de ruptura o erosión de las placas, donde se agregan trombos, y es la isquemia aguda y no la crónica en un miocardio condicionado, la que puede ocasionar infarto o muerte.

Aparentemente entonces, la anatomía coronaria supera a la isquemia como marcador de riesgo, pero en ninguno de los estudios randomizados, que excluyeron los grupos anatómicos de alto riesgo, la magnitud de la enfermedad anatómica favoreció la intervención.

Por este motivo, se ha hecho un gran esfuerzo por identificar las placas con riesgo de ruptura o vulnerables, que podrían guiar las intervenciones. Se ha encontrado que el mayor volumen de ateroesclerosis y de su componente lipídico, asociado a una capa fibrosa delgada, son los factores más importantes que se asocian al riesgo de ruptura. Sin embargo, la capacidad de predecir eventos de estas condiciones anatómicas es bajo ${ }^{21}$.

En suma, la estimación de riesgo basada en anatomía, fisiología o vulnerabilidad de las placas, en forma individual, tiene una baja capacidad predictiva de eventos, por lo que no serían fuertes determinantes de lesiones a intervenir. Así, en la enfermedad coronaria crónica, la predicción de eventos mayores atribuible a las características de las lesiones, alcanzaría a un $20 \%$ en 5 años para las severamente obstructivas ${ }^{6}$, a un $9 \%$ en 2 años en las que inducen isquemia significativa ${ }^{19}$, y a un $16-20 \%$ cuando tienen alta vulnerabilidad ${ }^{22}$. Cifras de riesgo que son similares a las obtenidas en pacientes intervenidos 
con angioplastía o cirugía.

Por lo tanto, como ninguno de los 3 aspectos individualmente es lo suficientemente predictor para decidir las intervenciones, se ha sugerido que la interacción de ellos podría permitir una mejor predicción. Así, en la actualidad se estudia el valor predictivo y la utilidad de intervención local, basado en combinaciones de estos aspectos. En este sentido son interesantes las nuevas observaciones sobre el significado del FFR. Estudios recientes han señalado que, con el mismo grado de estenosis angiográfica, aquéllas lesiones con mayor contenido lípidico y de magnitud de placa, además, las que tienen mayor remodelamiento positivo, se correlacionan con FFR bajo ${ }^{23-24}$. Es decir el FFR $<0,8$ podría reconocer las lesiones más vulnerables a eventos. Apunta en este sentido el hecho de que en el FAME-2 los pacientes con FFR > 0,8, en TMO, tuvieron muy baja incidencia de eventos. Sin embargo, esta medición aislada no es suficiente, porque como se ha señalado, su valor predictivo (+) también fue bajo.

Me atrevería a anticipar que aunque los estudios en curso puedan probar el concepto, difícilmente estas evaluaciones híbridas intracoronarias lograrán un impacto significativo en la reducción del riesgo global de esta enfermedad. Todas ellas requieren un examen invasivo, costoso y con riesgos, razonablemente no alcanzable para la mayoría de los pacientes con enfermedad coronaria crónica. Tal como sugiere el Registro Cardiovascular Nacional en Estados Unidos ${ }^{25}$, parece necesario un paso previo a la coronariografía. En este registro, el porcentaje de pacientes correctamente identificados con lesiones $>50 \%$ mediante los test no invasivos de isquemia, alcanzó solo entre un 44 a $45 \%$. Por otro lado, son interesantes los datos que tenemos sobre la utilidad del angio-TAC coronario. Mientras no hubo diferencias en el riesgo alejado usando angio-TAC o test no invasivos para guiar la coronariografía en el estudio PROMISE ${ }^{26}$, tanto en el estudio SCOT-HEART ${ }^{27}$ como en el $\mathrm{CATCH}^{28}$ se demostró significativa disminución de eventos cuando se hizo angio-TAC. Estos datos se suman al COURAGE que definen una mejor predicción con la anatomía. En parte estos resultados se explican por la capacidad de las imágenes en identificar a los individuos con anatomía de alto riesgo, pero si sumamos a las imágenes las capacidades técnicas que se le han ido incorporando al angio-TAC, como la medición no invasiva del FFR y la definición morfológica, hemodinámica y metabólica de las lesiones coronarias, probablemente en el futuro esta será la más amplia forma de evaluar el riesgo de los pacientes con enfermedad coronaria crónica y así guiar las intervenciones que requieran.

En el momento actual, mientras no haya más pruebas, en los pacientes con enfermedad coronaria crónica, seguimos decidiendo las intervenciones de acuerdo a las guías internacionales que recomiendan la revascularización cuando el tratamiento médico no logra un buen control de los síntomas o cuando se comprueba isquemia exten$\mathrm{sa}^{29-30}$, aunque como se ha dicho, esta premisa no se ha probado en presencia del tratamiento médico actual. Lo más próximo para apoyar o descartar esta recomendación será los resultados del estudio ISCHEMIA, cuyos primeros resultados se dispondrán el año 2017. 


\section{Referencias}

1.- KEELEY EC, BOURA JA, GRINES CL. Primary angioplasty versus intravenous thrombolytic therapy for acute myocardial infarction: a quantitative review of 23 randomized trials. Lancet 2003 ; 361: 13-20.

2.- FOX KA, CLAYTON TC, DAMMAN P, POCOCK SJ, DE WINTER RJ, TIJSSEN JG, et al. Long-term outcome of a routine versus selective invasive strategy in patients with nonST segment elevation acute coronary sindrome: a meta-analysis of individual patient data. J Am Coll Cardiol 2010; 55: 2435-45.

3.- YUSUF S, ZUCKER D, PEDUZZI P, FISHER LD, TAKARO T, KENNEDY JW, et al. Effect of coronary bypass graft surgery on survival: overview of 10 year results from randomized trial by Coronary Artery Bypass Graft Surgery Trialists Collaboration. Lancet 1994; 344: 563-70

4.- SMITH SC, BENJAMIN EJ, BONOW RO, et al. AHA/ACC secondary prevention and risk reduction therapy for patients with coronary and other atherosclerotic vascular disease: 2011 update: a guideline from the American Heart Association and American College of Cardiology Foundation. J Am Coll Cardiol 2011; 58: 2432-46.

5.- MIN JK, DUNNING A, LIN FY, ACHENBACH S, AL-MALLAH M, BUDOFF MJ, et al. Age and sex related differences in all-cause mortality risk based on coronary computed tomography angiography findings results from the International Multicenter CONFIRM (Coronary CT Angiography Evaluation for Clinical Outcomes: An International Multicenter Registry) of 23,854 patients without known coronary artery disease. J Am Coll Cardiol. 2011; 58: 849-860

6.- BODEN WE, O`ROURKE RA, TEO KK, et al, for the COURAGE Trial Research Group. Optimal medical therapy with or without PCI for stable coronary disease. N Engl J Med 2007; 356: 1503.16 .

7.- BARI 2D Study Group. A randomized trial of therapies for type 2 diabetes and coronary artery disease. N Engl J Med 2009; 360: 2503-15.

8.- VANZETTO G, ORMEZZANO O, FAGRET D, COMET M, DENIS B, MACHECOURT J. Long-term additive prognostic value of thallium- 201 myocardial perfusion imaging over clinical and exercise stress test in low to intermediate risk pa- tients: study in 1137 patients with 6-year follow-up. Circulation 1999; 100: 1521-7.

9.- CHANG SM, NABI F, XU J, PETERSON LE, ACHARI A, PRATT CM, et al. The coronary artery calcium score and stress myocardial perfu- sion imaging provide independent and comple- mentary prediction of cardiac risk. J Am Coll Cardiol 2009; 54: 1872-82.

10.- CORTIGIANI L, BORELLI L, RACITI M, BOVENZI F, PICANO E, MOLINARO S, et al. Prediction of mortality by stress echocardiography in 2835 diabetic and 11305 nondiabetic patients. Circ Cardiovasc Imaging 2015; 8: e002757.

11.- JOHNSON NP, TÓTH GG, LAI D, ZHU H, AÇAR G, AGOSTONI P, et al. Prognostic value of fractional flow reserve: linking physiology severity to clinical outcomes. J Am Coll Cardiol 2014; 64: 1641-54.

12.- HUEB W, LOPES N, GERSH BJ, SOARES PR, RIBEIRO EE, PEREIRA AC, et al. Ten-year follow-up survival of the Medicine, Angioplasty or Surgery Study (MASS II): a randomized controlled clinical trial of 3 therapeutic strategies for multivessel coronary artery disease. Circulation 2010;122:949-57.

13.- JEREMIAS A, KAUL S, ROSENGART TK, GRUBERG L, BROWN DL. The impact of revascularization on mortality in patients with non acute coronary artery disease. Am J Med 2009;122:152-61.

14.- SHAW LJ, BERMAN DS, MARON DJ, MANCINI GB, HAYES SW, HARTIGAN PM, et al, for the COURAGE Investigators. Optimal medical therapy with or without percutaneous coronary intervention to reduce ischemic burden: results from the COURAGE trial Nuclear Substudy: Circulation 2008; 117: 1283-91

15.- MANCINI GB, HARTIGAN PM, SHAW LJ, BERMAN DS, HAYES SW, BATES ER, et al. Predicting outcome in the COURAGE trial: coronary anatomy versus ischemia: $\mathrm{J}$ Am Coll Cardiol Intv 2014; 7: 195-201.

16.- SHAW LJ, CERQUEIRA MD, BROOKS MM, ALTHOUSE AD, SANSING VV, BELLER GA, et al. Impact of left ventricular function and the extent of ischemia and scar by stress myocardial perfusion imaging on prognosis and therapeutic 
risk reduction in diabetic patients with coronary artery disease from the BARI-2D trial. J Nucl Cardiol 2012; 19: 658-69

17.- PANZA JA, HOLLY TA, ASCH FM, SHE L, PELLIKKA PA, VELAZQUEZ EJ, et al. Inducible myocardial ischemia and outcomes in patients with coronary artery disease and left ventricular dysfunction. J Am Coll Cardiol 2013; 61: 1860-70

18.- PIJLS NH, DE BRUYNE B, PEELS K, VAN DER VOORT PH, BONNIER HJ, BARTUNEK J KOOLEN JJ, et al. Measurement of fractional flow reserve to asses the functional severity of coronary artery stenosis. N Engl J Med 1996; 334: 1703-1708

19.- DE BRUYNE B, PIJLS NH, KALESAN B, BARBATO E, TONINO PA, PIROTH Z, et al. Fractional flow reserve-guided PCI versus medical therapy in stable coronary disease. N Engl J Med 2012; 367: 991-1001.

20.- LIBBY P. Mechanisms of acute coronary syndromes and their implications for therapy. N Engl J Med 2013; 368: 2004-13

21.- STONE GW, MAEHARA A, LANSKY AJ, DE BRUYNE B, CRISTEA E, MINTZ GS, et al. PROSPECT Investigators. A prospective natural history study of coronary atherosclerosis. N Engl J Med 2011; 364: 226-235

22.- MOTOYAMA S, ITO H, SARAI M, KONDO T, KAWAI H, NAGAHARA Y, et al. Plaque characterization by coronary computed tomography angiography and the likelihood of acute coronary events in mid-term follow-up. J Am Coll Cardiol. 2015; 66: 337-346

23.- PARK HB, HEO R, Ó HARTAIGH B, CHO I, GRANSAR H, NAKAZATO R, et al. Atheroesclerotic plaque characteristics by $\mathrm{CT}$ angiography identify coronary lesions that cause isquemia: a direct comparison to fractional flow reserve: JACC Cardiovasc Imaging 2015; 8: 1-10
24.- GAUR S, ØVREHUS KA, DEY D, LEIPSIC J, BØTKER HE, JENSEN JM, et al. Coronary plaque quantification and fractional flow reserve by coronary computed tomography angiography identify ischaemia-causing lesions. Eur Heart J 2016; 37: 1220-1227

25. PATEL MR, DAI D, HERNANDEZ AF, DOUGLAS PS, MESSENGER J, GARRATT KN, et al. Prevalence and predictors of nonobstructive coronary artery disease identified with coronary angiography in contemporary clinical practice. Am Heart J. 2014; 167: 846-852.

26.- DOUGLAS PS, HOFFMANN U, PATEL MR, MARK DB, AL-KHALIDI HR, CAVANAUGH B, et al; PROMISE Investigators. Outcomes of anatomical versus functional testing for coronary artery disease. N Engl J Med. 2015; 372: 1291-1300

27.- LINDE JJ, HOVE JD, SØRGAARD M, KELBÆK H, JENSEN GB, KÜHL JT, et al. Long-term clinical impact of coronary CT angiography in patients with recent acute-onset chest pain: The Randomized Controlled CATCH Trial. JACC Cardiovasc Imaging. 2015; 8: 1404-1413. McKavanagh P,

28. WILLIAMS MC, HUNTER A, SHAH AS, ASSI V, LEWIS S, SMITH J, et al; SCOT-HEART Investigators. Use of Coronary Computed Tomographic Angiography to Guide Management of Patients With Coronary Disease. J Am Coll Cardiol. 2016; 67: 1759- 1768 .

29.- WINDECKER S, KOLH P, ALFONSO F, COLLET JP, CREMER J, FALK V, et al. 2014 ESC/EATS guidelines on myocardial revascularization. Eur Heart J 2014; 35: 2541-619.

30.- PATEL MR, DEHMER GJ, HIRSHFELD JW, SMITH PK, SPERTUS JA. CCF/SCAI/STS/AATS/AHA/ASNC/HFSA/ SCCT 2012 appropriate use criteria for coronary revascularization focused update. J Am Coll Cardiol 2012; 59: 857-81. 